\title{
Lymphocytic-Plasmacytic Enteritis as a Cause of Long-Lasting Weight Loss and Recurrent Colic in an Elderly Horse
}

\author{
Catarina João Gil de Barros ${ }^{1}$, Luís Nuno Moreirão Cerca Monteiro de Oliveira² ${ }^{2}$ Ricardo Brás \\ Campos $^{3}$ and Pedro Pinto-Bravo ${ }^{4 *}$ \\ ${ }^{1}$ Equicare Veterinary Services; Rua Dr. Luís Brás de Abreu 13, 2esq, 3810-100 Aveiro, Portugal
}

${ }^{2}$ Faculty of Veterinary Medicine, Vasco da Gama University School; Largo da Amoreira 34, 5150-014 Almendra, Portugal

3Faculty of Veterinary Medicine, Vasco da Gama University School, Equicare Veterinary Services; Rua Álvaro Cunhal 22, 3040 350 Coimbra, Portugal

${ }^{4}$ CERNAS (Research Center for Natural Resources, Environment and Society), Polytechnic Institute of Coimbra, Coimbra, Portugal

*Corresponding author: Pedro Pinto-Bravo, CERNAS (Research Center for Natural Resources, Environment and Society),

Polytechnic Institute of Coimbra, Coimbra, Portugal

\section{ARTICLE INFO}

Received: 慧 February 11, 2021

Published: 㓞 February 17, 2021

Citation: Catarina João Gil de Barros, Luís Nuno Moreirão Cerca Monteiro de Oliveira, Ricardo Brás Campos, Pedro Pinto-Bravo. Lymphocytic-Plasmacytic Enteritis as a Cause of Long-Lasting Weight Loss and Recurrent Colic in an Elderly Horse. Biomed J Sci \& Tech Res 34(1)-2021. BJSTR. MS.ID.005485

\begin{abstract}
Lymphocytic-Plasmacytic Enteritis (LPE) is an uncommon cause of chronic inflammatory bowel disease in horses. The proliferative inflammatory cell infiltrate results in diffuse mucosal inflammation and impaired nutrients absorption, leading to chronic weight loss, recurrent abdominal pain and hypoproteinemia. This article reports the case of a 25-year-old horse with LPE causing long-lasting severe cachexia and recurrent colic episodes. A positive response to diet changes and medical treatment with corticosteroids was observed during a short period of time. However, relapse of recurrent colic episodes refractory to medical treatment determined the euthanasia of the horse.
\end{abstract}

Keywords: Horse; Chronic Weight Loss; Recurrent Colic; Small Intestinal Malabsorption Syndrome; Lymphocytic-Plasmacytic Enterocolitis

Abbreviations: CIBD: Chronic Inflammatory Bowel Disease; EPE: Equine Proliferative Enteritis; LPE: Lymphocytic Plasmacytic Enteritis; MEED: Multisystemic Eosinophilic Epitheliotrophic Disease; OGTT: Oral Glucose Tolerance Test

\section{Introduction}

Intestinal malabsorption implies impaired digestive and absorptive processes due to functional or structural disorders of the small intestinal tract and/or related organs, such as pancreas, liver and biliary tract. Primarily clinical signs observed in affected horses are chronic wasting and poor body condition. Other signs may include recurrent colic, diarrhoea, anorexia, lethargy, fever, edema and skin lesions [1]. Malabsorption can be diagnosed by performing an Oral Glucose Tolerance Test (OGTT). Horses with total malabsorption reveal an increase of blood glucose less than $15 \%$ over the resting level 120 minutes after administering $1 \mathrm{~g}$ of glucose per $\mathrm{kg}$ bwt as a $20 \%$ solution by nasogastric intubation [2]. Chronic Inflammatory Bowel Diseases (CIBD) are the most common cause of malabsorption in adult horses. Other causes include extensive small intestinal resection, enteric infections (Mycobacterium tuberculosis, Aspergillus fumigatus, Lawsonia intracellularis), parasitic infections (cyathostominosis), intestinal lymphoma and intestinal fibrosis [3].

CIBD collectively refers to a group of proliferative or inflammatory intestinal diseases, classified according to the cellular infiltrate type. Other frequent pathological changes within the small intestinal mucosa include villous stunting, fusion and deformation [4]. Differentiation between different causes of CIBD relies upon histopathological examination. In horses, main proliferative diseases causing CIBD are granulomatous enteritis, 
idiopathic eosinophilic enterocolitis, multi systemic eosinophilic epitheliotropic disease (MEED) and lymphocytic-plasmacytic enterocolitis [5]. In this paper, we report a case of LymphocyticPlasmacytic Enteritis (LPE) in an elderly horse, presenting longlasting severe cachexia and recurrent colic episodes [6].

\section{Case history}

A 25 year-old crossbreed gelding was brought to the clinic for colic examination. Anamnesis included long-lasting weight loss and recurrent colic episodes responsive to medical therapy. The horse was properly vaccinated and dewormed. Current feeding plan included low quality hay and regular grain, both fed twice daily. General clinical examination revealed a 1/9 body condition score, tachycardia and tachypnea (72 bpm and $32 \mathrm{rpm}$, respectively), normal rectal temperature and generalized hypomotility [7]. Blood examination revealed hipoproteinemia (50 g/L) with no other abnormalities. Rectal palpation revealed distended, fluid-filled and thickened-wall small intestines. Moderate gas distention was palpable at the level of the left dorsal colon and cecum. There were no signs of displacement. Sand test was performed and deemed negative. On rectal ultrasound, small intestinal wall thickness was accessed, with identification of normal $(<0,4 \mathrm{~mm})$ and thickened $(0,7$ up to $1,2 \mathrm{~mm})$ loops.

Ventral abdomen ultrasound revealed an increased amount of anechogenic free fluid. Abdominocentesis was performed, and a strong yellow mildly turbid fluid was collected, with increased total proteins (48 g/L). Nasogastric intubation allowed to recuperate $4 \mathrm{~L}$ of spontaneous reflux, followed by stomach flushing and hydrotherapy with 3 liters of water and 300 grams of magnesium sulfate. Fluid therapy was performed with 10 liters of Ringer Lactate, 1 liter of 7,5\% Sodium Chloride and $800 \mathrm{ml}$ of a balanced amino acids, vitamins and minerals solution (Duphalyte $($ ) [8]. Non-steroidal anti-inflammatory drug flunixin meglumine was administered once $(2,2 \mathrm{mg} / \mathrm{kg}$ IV). Antibiotic therapy with penicillin-streptomycin (10 mg/kg IM SID) was maintained for 5 days. During the following hours, the horse remained comfortable, with no reflux production and re-establishment of normal gastrointestinal motility was detected. Due to financial reasons, OGTT and further exams (such as gastroscopy and intestinal biopsies) were not performed. Although, due to the high suspicion of inflammatory bowel disease, treatment with corticosteroids and diet changes were recommended. Intramuscular dexamethasone sodium phosphate injections were administered at a dosage of 0,1 $\mathrm{mg} / \mathrm{kg}$ SID for 7 days followed by $0,05 \mathrm{mg} / \mathrm{kg}$ SID for 7 days $[9,10]$.

The horse was kept in short grass pasture over the day, receiving small amounts of soaked hay multiple times a day. Bran mash was also provided twice daily. During the course of treatment and following three months the horse remained comfortable with appetite improvement, although no remarkable changes in body condition were observed. Colic episode relapses started to occur, becoming progressively less responsive to medical therapy.
Based on horse's clinical condition and for well-being reasons, euthanasia was performed with owner consentment. Postmortem examination was immediately performed. Thickened-wall jejunum was macroscopically identified in about $80 \%$ of its full length, followed by samples collection and preservation in $10 \%$ formol. Histopathological analysis revealed the presence of chronic LPE, with severe villi and mucosa glands atrophy (Figures $1 \& 2$ ).

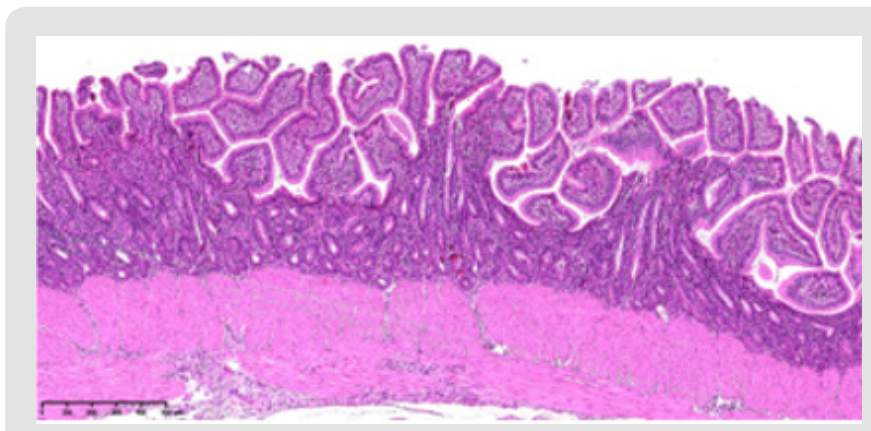

Figure 1: Representative histopathological images of the predominant lymphocytic-plasmacytic inflammatory infiltrate of the jejunum mucosa, crypt glands hyperplasia and marked villous atrophy.

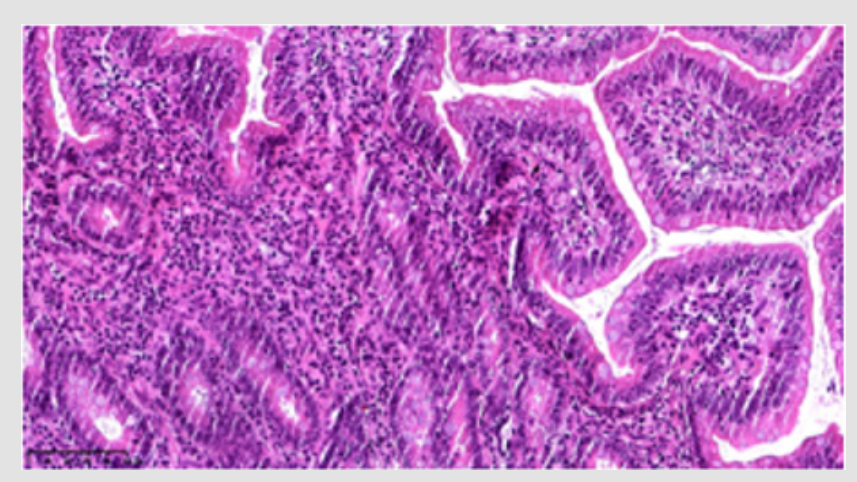

Figure 2: Hematoxylin-eosin staining.

\section{Discussion}

CIBD, the main cause of malabsorption syndrome in horses, associate with gastrointestinal tract dysfunction due to mucosa and/or submucosa infiltration with different inflammatory cell types, such as eosinophils, basophils, lymphocytes and plasma cells. CIBD is frequently associated with protein-losing enteropathy due to compromising of gastrointestinal wall integrity, demonstrating poor prognosis. According to a recent retrospective study, main clinical signs of CIBD in horses include weight loss $(78,2 \%)$, recurrent colic episodes $(28,2 \%)$, lethargy $(21,8 \%)$ and diarrhea $(14,1 \%)$. LPE is an uncommon cause of CIBD in horses, with no age, breed, diet or sex predisposing factors associated (Schumacher et al., 2000). In horses, and although further studies are required, LPE may represent a nonspecific intestinal immune response to bacterial, viral, parasitic and dietary antigens that cause epithelial damage. Additionally, as the most common type of idiopathic CIBD in dogs, LPE is suspected to represent an early stage of lymphoma, and differentiation between those two conditions can 
be challenging. Definitive diagnosis of CIBD can only be obtained by histopathological examination of intestinal full thickness biopsies obtained by exploratory laparotomy, laparoscopy or during postmortem examination. The 25-year-old horse presented in this paper was diagnosed with LPE by histopathology, also revealing some similarities to Equine Proliferative Enteritis (EPE) caused by L. intracellularis. Although no molecular or serological diagnostic tests were performed, EPE commonly affects foals less than 12 months of age.

Treatment for equine CIBD is based on dietary adjustments and corticosteroid therapy with prednisolone or dexamethasone, with medical therapy being necessary for a period rarely less than 3 months. In the presented case, due to financial reasons medical treatment was performed only for two weeks, although a positive response was observed, with appetite improvement and absence of colic signs during the following 3 months. Although results from previous studies vary, LPE is associated with long-term poor prognosis. Degree of villous atrophy seems to be a determining factor on prognosis establishment. Chronic LPE is reported as an uncommon cause of malabsorption syndrome in horses. In this case report, we present the case of an elderly horse with malabsorption syndrome caused by a well-advanced LPE, with more than 5 years of weight loss and recurrent colic history. Medical therapy and dietary changes were tried under limited conditions, and despite we found a positive response in the following months, relapse of recurrent colic episodes refractory to medical treatment determined the euthanasia of the horse.

\section{Declaration}

The authors have declared no competing interests.

ISSN: 2574-1241

DOI: 10.26717/BJSTR.2021.34.005485

Pedro Pinto-Bravo. Biomed J Sci \& Tech Res

This work is licensed under Creative Commons Attribution 4.0 License

Submission Link: https://biomedres.us/submit-manuscript.php

\section{Acknowledgement}

Wewant to thank the team atthe Veterinary Anatomopathological Analysis Laboratory VetPat ${ }^{\circledR}$ for performing the histopathological analysis of the intestinal samples.

\section{References}

1. Boshuizen B, Ploeg M, Dewulf J, Klooster S, de Bruijn M (2018) Inflammatory bowel disease (IBD) in horses: a retrospective study exploring the value of different diagnostic approaches. BMC Vet Res 14(1): 21.

2. Kaikkonen R, Niinistö K, Sykes B, Anttila M, Sankari S (2014) Diagnostic evaluation and short-term outcome as indicators of long-term prognosis in horses with findings suggestive of inflammatory bowel disease treated with corticosteroids and anthelmintics. Acta Vet Scand 56(35): 1-6.

3. Kalck KA (2009) Inflammatory bowel disease in horses. Vet Clin North Am Equine Pract 25(2): 303-315.

4. Kemper DL, Perkins GA, Schumacher J, Edwards JF, Valentine BA (2000) Equine lymphocytic-plasmacytic enterocolitis: a retrospective study of 14 cases. Equine Vet J 32: 108-112.

5. Mair TS, Pearson GR, Divers TJ (2006) Malabsorption syndromes in the horse. Equine Vet Educ 18(6): 299-308.

6. Mair TS, Sherlock CE, Fews D, Harley R, Pearson GR (2016) Idiopathic fibrosis of the tunica muscularis of the large intestine in five horses with colic. J Comp Path 154(2-3): 231-234.

7. Pusterla N, Gebhart CJ (2013) Equine proliferative enteropathy - a review of recent developments. Equine Vet J 45(4): 403-409.

8. Schumacher J, Edwards JF, Cohen ND (2000) Chronic idiopathic inflammatory bowel diseases of the horse. J Vet Intern Med 14(3): 258265.

9. Siwinska N, Zak A, Slowikowska M, Borowicz H, Nowak M (2017) Progressive lymphocytic-plasmacytic enteritis in a horse. Pferdeheilkunde 33(5): 452-456.

10. Stewart HL, Engiles JB, Stefanovski D, Southwood L (2018) Clinical and intestinal histologic features of horses treated for recurrent colic: 66 cases (2006-2015). J Am Vet Med Assoc 252(10): 1279-1288.

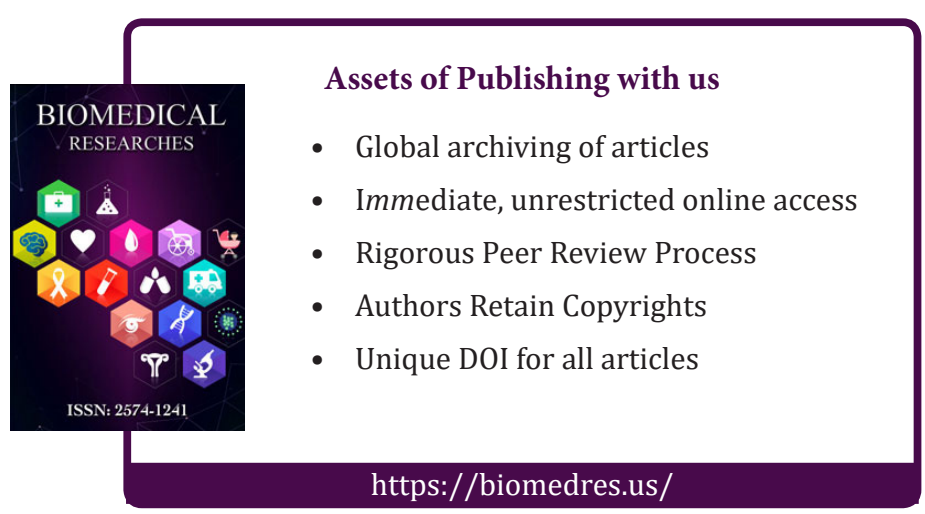

